\title{
Relaciones del Plexo Braquial con la Segunda Parte de la Arteria Axilar
}

\author{
Brachial Plexus Relationships whith the Second Part of Axillary Artery \\ "Luiz Carlos Buarque de Gusmão; "Célio Fernando de Sousa Rodrigues; "* Moana Vergetti Malta; *** Klebiana Santos; "** \\ Luiz Victor Maia Loureiro; ${ }^{* * *}$ Maria Helena Luz Machado; ${ }^{* * *}$ Pablo Coutinho Malheiros; ${ }^{* * * *}$ Shyrlene Santana Santos; \\ **** Ulisses Vitor Pereira Neto \& **** Yara Gomes Falcão Almeida
}

GUSMÃO, L. C. B.; SOUSA-RODRIGUES, C. F.; MALTA, V. M.; SANTOS, K.; LOUREIRO, L. V. M.; MACHADO, M. H. L.; MALHEIROS, P. C.; SANTOS, S. S.; PEREIRA NETO, U. V. \& ALMEIDA, F. Y. G. Relaciones del plexo braquial con la segunda parte de la arteria axilar. Int. J. Morphol., 23(2):129-132, 2005.

RESUMEN: Analizamos 62 axilas, 32 derechas y 30 izquierdas de cadáveres humanos, con el objetivo de hacer un estudio detallado de las relaciones topográficas de los cordones del plexo braquial (PB) en relación a la 2 ${ }^{\mathrm{a}}$ parte de la arteria axilar (Aa) (parte retropectoral de la axila). Notamos que algunos cordones del PB pierden su identidad a lo largo de dicha porción, por este motivo, el análisis de ellos fue hecha en el comienzo de la región retropectoral y se limitó a los casos en que se presentaban al mismo tiempo los tres cordones (36 casos, 58\%). Se comprobó que en el 27,42\% de los casos, los cordones del PB circundaban la 2a parte de la Aa y estaban situados en conformidad con sus nombres - lateral (CL), medial (CM) y posterior (CP) - siendo ésta la disposición más citada en la literatura. En 16,13\% de los casos, se observó el CM y el CP dispuestos posteriormente a la Aa, estando el CL lateral. En 6,46\% de los casos, el CP estaba ubicado postero-lateral, el CM estaba posterior y el CL se ubicaba lateralmente a la Aa. En 3,23\% de los casos, el CL y el CM estaban situados anteriormente, y el CP posteriormente a la Aa; en el mismo porcentaje todos los cordones del PB estaban ubicados lateralmente a la Aa. Sólo en el 1,62\%, fueron encontrados los CM y CP relacionados con la Aa conforme a sus nombres, y el CL ubicado anteriormente a la Aa. En la mayoría de los casos donde los cordones estaban presentes, éstos se ubicaban de modo diferente a como se presenta en la literatura analizada.

PALABRAS CLAVE: Axila; Arteria axilar; Plexo braquial.

\section{INTRODUCCIÓN}

La inervación motora y sensitiva de los miembros superiores se realiza a través del plexo braquial (PB), formado por la unión de los ramos ventrales de los cuatro nervios cervicales inferiores y la mayor parte del ramo ventral del primer nervio torácico. Los troncos formados se bifurcan posteriormente a la clavícula, en divisiones anteriores y posteriores, formando los cordones lateral, medial y posterior. En las axilas, los cordones tienden a envolver la segunda parte de la arteria axilar (Aa), relacionados con ella en conformidad con sus nombres - lateral, medial y posterior siendo ésta la ubicación más aludida en la literatura anatómica (Buchanan, 1919; Dwight, 1919; Davis, 1921; Reding, 1921; Grant, 1948; Rouvière, 1959; Lockhart et al., 1965;
Hollinshead, 1966; Spalteholz, 1967; Gardner et al., 1975; Snell, 1981; Hamilton, 1982; Raj, 1985; Weisse, 1888; Latarjet \& Liard, 1989; Williams et al., 1989; Woodburne, 1989; Moore, 1994 y Dângelo \& Fattini, 1995).

Los datos referentes a las relaciones del PB con la $2^{\mathrm{a}}$ parte de la Aa, existentes en la literatura estudiada son escasos en detalles. Las ubicaciones de los cordones son descritas, en la mayoría de los casos, sin determinar con qué parte de la arteria ocurren.

A causa de las discrepancias verificadas en la literatura analizada, de los diferentes casos encontrados en las

\footnotetext{
* Doctores en Anatomía Humana; Departamento de Morfología - Universidad Federal de Alagoas - Alagoas, Brasil.

* Monitora de Anatomía; Bolsista de Iniciacion Científica del CNPq, Brasil.

**** Estudiantes de Medicina de la Universidade Federal de Alagoas, Brasil.
} 
GUSMÃO, L. C. B.; SOUSA-RODRIGUES, C. F.; MALTA, V. M.; SANTOS, K.; LOUREIRO, L. V. M.; MACHADO, M. H. L.; MALHEIROS, P. C.; SANTOS, S. S.; PEREIRA NETO, U. V. \& ALMEIDA, F. Y. G.

disecciones de laboratorio y de la importancia de conocer la ubicación de los componentes del paquete vásculo-nervioso de la región, principalmente durante las intervenciones quirúrgicas, nos propusimos efectuar un estudio más detallado de las relaciones topográficas de los cordones del PB en relación con la $2^{\mathrm{a}}$ parte de la Aa, y así suministrar datos más exactos y correctos sobre la ubicación de los cordones nerviosos en esta parte de la axila.

\section{MATERIAL Y MÉTODO}

Fueron analizadas 62 axilas de cadáveres humanos, de ambos sexos y de diferentes edades y etnias, fijados en formaldehído al 10\%, pertenecientes a los Departamentos de Morfología de la Universidad Federal de Alagoas, de la Universidad Tiradentes de Sergipe y de la Escuela de Ciencias Médicas de Alagoas, Brasil.

Con los cuerpos en decúbito dorsal, los músculos pectorales mayor y menor fueron desinsertados y rebatidos posibilitando la identificación de la Aa y del PB. Para la determinación de la $2^{\mathrm{a}}$ parte de la axila (sitio correspondiente a la proyección del músculo pectoral menor), el músculo pectoral menor fue colocado en su posición original y fijado con dos alfileres, delimitándose la $2^{\mathrm{a}}$ parte de la Aa. Así fueron observadas y registradas las relaciones entre los cordones del PB y la Aa.
En 17 casos (27,42\%), el cordón lateral (CL) se relacionó lateralmente con la Aa, el cordón medial (CM), medialmente, y el cordón posterior $(\mathrm{CP})$, posteriormente, conforme se puede observar en la Fig. 1 Tipo I.

En 10 casos $(16,13 \%)$ se encontraban los CM y CP posteriores a la Aa, y el CL se localizaba lateralmente, como se puede observar en la Fig. 1 Tipo II.

En 4 casos $(6,46 \%)$, el CP se ubicaba postero-lateral, el CM se encontraba posterior y el CL se presentaba lateralmente a la Aa. Fig. 1 Tipo III.

En 2 casos $(3,23 \%)$, el CL y el CM estaban anteriormente a la $\mathrm{Aa}$, y el CP se ubicaba posteriormente a la misma, como se puede observar en la Fig. 1 Tipo IV.

En 2 casos, todos los cordones del PB se ubicaban lateralmente a la Aa, persistiendo, así en la misma porción en que se encontraban en la $1^{\mathrm{a}}$ parte de la axila, conforme a lo presentado en la Fig. 1 Tipo V.

Solamente en un caso $(1,62 \%)$ se encontró el CM y el CP relacionados con la Aa en conformidad con sus nombres, y el CL ubicado anteriormente a la referida arteria. Fig. 1 Tipo VI.

\section{RESULTADOS}

El examen de las axilas mostró que en 26 casos (42\%) algunos cordones del PB perdían su identidad a lo largo de la parte retropectoral de la axila. En esos casos, los respectivos cordones se bifurcaban en sus ramos terminales desde la región suprapectoral. Así, el análisis de las relaciones de los cordones con la $2^{\mathrm{a}}$ parte de la Aa fue hecha en el comienzo de la región retropectoral y se limitó a los casos en que estaban presentes al mismo tiempo los tres cordones, 36 casos (58\%).

Analizándose las 36 axilas que presentaban los tres cordones del PB, se constató la existencia de 6 tipos diferentes de ubicaciones de los cordones. Fig. 1.

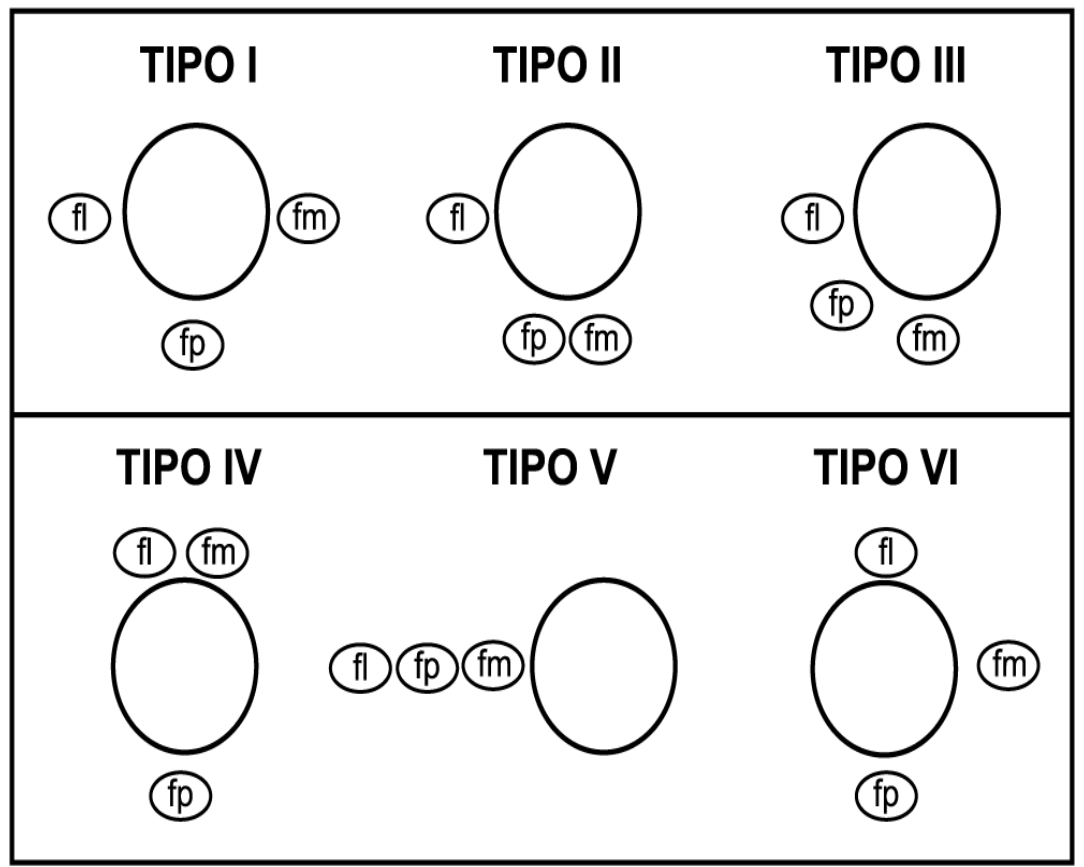

Fig. 1. Tipos diferentes de ubicaciones de los cordones del plexo braquial. 
Relaciones del plexo braquial con la segunda parte de la arteria axilar. Int. J. Morphol., 23(2):129-132, 2005.

\section{DISCUSIÓN}

El estudio reveló que los datos concernientes a las relaciones de los cordones del PB con la $2^{\mathrm{a}}$ parte de la Aa encontrados en la literatura investigada, son pobres en detalles y disconformes entre sí. La mayor parte de la literatura investigada (Buchanan; Dwight; Davis; Reding; Grant; Rouvière; Lockhart et al.; Hollinshead; Spalteholz; Gardner et al.; Snell; Hamilton; Raj; Weisse; Latarjet \& Liard; Williams et al.; Woodburne; Moore y Dângelo \& Fattini) se limita a describir la ubicación de los cordones, en relación con la $2^{\mathrm{a}}$ parte de la Aa, de conformidad con sus nombres - medial, lateral y posterior - siendo ésta la disposición encontrada solamente en 17 casos $(27,42 \%)$.

El número relevante de 10 casos $(16,13 \%)$ muestra una ubicación poco relatada en la literatura investigada, en la cual los CM y CP están posteriores a la Aa, y el CL se localiza lateralmente (Anson, 1963; Putz \& Pabst, 2000), conforme vemos en la Fig. 1, Tipo II.

En su estudio, Adachi (1928) relata una disposición en la cual todos los cordones están localizados lateralmente a la $2^{\mathrm{a}}$ parte de la Aa, manteniéndose así, en la misma posición en que se encuentran en la $1^{\text {a }}$ parte de la axila y de la Aa. Encontramos en este estudio, 2 casos $(3,23 \%)$ donde los cordones están en la disposición relatada anteriormente. La ubicación medial de todos los cordones del PB en relación con la $2^{\mathrm{a}}$ parte de la Aa, conforme describe Gusmão (1992), no ha sido identificada en ninguna de las axilas estudiadas.
Destacamos que las otras ubicaciones encontradas en nuestro estudio, 33 casos $(53,22 \%)$, no han sido relatadas por ninguno de los autores investigados. Las diferentes variaciones parecen ser muy importantes, pues son ellas la causa de ciertos fracasos en los tratamientos quirúrgicos de las lesiones del plexo braquial (Fazan et al., 2003).

Son poco frecuentes las descripciones en la literatura que aluden la posibilidad de ausencia de algún cordón del PB en la región retropectoral de la axila, siendo que, en este caso, la $2^{a}$ parte de la Aa se relaciona con los ramos terminales de los cordones ausentes, como ha sido descrito por Testut \& Jacob (1932). Consideramos muy alto el porcentaje de 26 casos (42\%) en donde ocurre la ausencia de por lo menos un cordón en la región retropectoral, siendo este hallazgo de gran importancia, en las intervenciones quirúrgicas que se realizan en la región axilar.

En este estudio, observamos variaciones de importancia en cuanto a la ubicación de los cordones del $\mathrm{PB}$ en relación con la Aa, posibilidad de ausencia de los cordones en la porción retropectoral de la axila y relación de los ramos terminales de los cordones ausentes con la $2^{\mathrm{a}}$ parte de la Aa. Es importante dar a conocer estos datos para incrementar el conocimiento anatómico sobre la ubicación de los cordones del PB en relación con la Aa y servir de soporte morfológico para los procedimientos quirúrgicos a realizarse en la región axilar.

GUSMÃO, L. C. B.; SOUSA-RODRIGUES, C. F.; MALTA, V. M.; SANTOS, K.; LOUREIRO, L. V. M.; MACHADO, M. H. L.; MALHEIROS, P. C.; SANTOS, S. S.; PEREIRA NETO, U. V. \& ALMEIDA, F. Y. G. Brachial plexus relationships whith the second part of axillary artery. Int. J. Morphol., 23(2):129-132, 2005.

SUMMARY: 62 axillas, 32 on the right upper limb and 30 on the left one were analyzed aiming a more detailed study of the topographic relationship between the brachial plexus' (BP) cords and the second part of the axillary artery (Aa). Once the second part of the Aa was determined (retropectoral part of the axilla) the authors observed that some of the BP's cords lost their identity during the length of the above-mentioned part of the axilla, so, the analysis of these cords and their relationship with the Aa was effected on the beginning of the retropectoral region. It was verified that in $27,42 \%$ of the cases, PB's cords surrounded the 2 nd part of the Aa positioned according to their names - lateral cord (LC), medial cord (MC) and posterior cord (PC) - being this the most mentioned disposition in literature. In 16,13\% of the cases, it was observed that MC and PC were disposed posteriorly to the Aa, while LC was situated laterally to the artery. In 3,23\% of the cases, LC and MC were found anteriorly, and PC posteriorly to the Aa; in the same percentage of cases, all the cords were situated laterally to the Aa. Only in 1,62\%, MC and PC were situated according to their names, and the LC was anterior to the Aa. The majority of cases in which the cords were present, they disposed themselves differently of what the analyzed literature shows.

KEY WORDS: Axilla; Axillary Artery; Brachial Plexus. 
GUSMÃO, L. C. B.; SOUSA-RODRIGUES, C. F.; MALTA, V. M.; SANTOS, K.; LOUREIRO, L. V. M.; MACHADO, M. H. L.; MALHEIROS, P. C.; SANTOS, S. S.; PEREIRA NETO, U. V. \& ALMEIDA, F. Y. G.

\section{REFERENCIAS BIBLIOGRÁFICAS}

Adachi, B. Anatomia dos japoneses: o sistema arterial dos japoneses. Kyoto, Universidade Real-Japonesa de Kyoto, 1928. V. 1.

Anson, B. J. An atlas of human anatomy. 2. ed. Philadelphia, WB. Saunder, 1963.

Buchanan, A. M. Manual of anatomy. 4. ed. St. Louis, 1919, V. 1.

Dângelo, J. G. \& Fattini, C. A. Anatomia humana sistêmica e segmentar. 2. ed. São Paulo, Atheneu, 1995.

Davis, G. G. Applied anatomy. 5. ed. London, J.B. Leppincott, 1921.

Dwight, T. et al. Human anatomy. 7. ed. Philadelphia, J.B. Leppincott, 1919. V. 1.

Fazan, V. P. S.; Amadeu, A. S.; Caleffi, A. L. \& Filho, O. A.R. Brachial plexus variations in its formation and main branches. Acta Cir. Bras., 18:14-9, 2003.

Gardner, E.; Gray, D. J. \& O'rahilly, R. Anatomia. 4. ed. Rio de Janeiro, Guanabara Koogan, 1975.

Grant, J. C. B. A Method of Anatomy. 4. ed. Baltimore, Williams and Wilkins, 1948.

Gusmão, L. C. B. Anatomical study of the accessory axillary vein. J. Clin. Anatomy, 14:131-6, 1992.

Hamilton, W. J. Tratado de anatomia humana. 2. ed. Rio de Janeiro, Interamericana, 1982.

Hollinshead, W. H. Anatomia humana. Rosario, Harper \& Row, 1966.

Latarjet, M. \& Liard, A. R. Anatomia humana. 2. ed. México, Panamericana, 1989.

Lockhart, R. D.; Hamilton, G. F. \& Fyfe, F. W. Anatomia humana. $1^{a}$ ed. México, Interamericana, 1965.

Moore, Keith L. Anatomia orientada para a clínica. 3. ed. Rio de Janeiro, Guanabara Koogan, 1994.

Putz, R. \& Pabst, R. Atlas de anatomia humana Sobotta. 21. ed. Rio de Janeiro, Guanabara Koogan, 2000. V. 1.
Raj, P. Prithvi. Handbook of regional anesthesia. Nova Iorque, Churchill Livingstone, 1985.

Reding, M. D’anesthésie régionale du member supérieur. Mercredi, 1921.

Rouvière, M. Anatomie humaine descriptive et topographique. 8. ed. Paris, Maison, 1959 V. 1.

Snell, R. S. Anatomia. 2. ed. Rio de Janeiro, Médica e Científica, 1981.

Spalteholz, W. Atlas de anatomia humana. 3. ed. Barcelona, Labor, 1967.

Testut, L. \& Jacob, O. Tratado de anatomia topográfica com aplicaciones médico-quirurgicas. 5. ed. Barcelona, Salvat, 1932. V. 2.

Weisse, F. D. Pratical human anatomy. 3. ed. 1888. V. 3.

Williams, P. L. et al. Gray anatomia. 37. ed. Rio de Janeiro, Guanabara Koogan, 1989. V. 2.

Woodburne, R.T. Anatomia Humana. 6. ed. Rio de Janeiro, Guanabara Koogan, 1984.

Dirección para correspondencia:

Prof. Dr. Luiz Carlos Buarque de Gusmão

Condominio Aldebaran ALFA - Quadra F - N 08

Tabuleiro dos Martins

CEP:57080-900

Maceió - Alagoas

BRASIL

Recibido : 27-12-2004

Aceptado: 11-05-2005 